\title{
LARYNGEAL SYMPTOMS
}

WHICH RESULT FROM THE

\section{PRESSURE OF ANEURISMAL AND OTHER TUMOURS UPON THE VAGUS AND RECURRENT NERVES.}

\author{
BY \\ GEORGE JOHNSON, M.D., F.R.S., \\ PHISICIAN TO KING's COLLEGE HOSPITAL.
}

(Received November 10th-Read December 8th, 1874.)

Amongst the practical gains which have resulted from the use of the laryngoscope one of the greatest has been the increased facility which the inspection of the living larynx has afforded for investigating the results of disease implicating the vagus and recurrent nerves.

The main object of the present communication is first to demonstrate and then to explain the fact that bilateral spasm and bilateral palsy of the intrinsic muscles of the larynx may result from the pressure of an aneurism or other tumour on the vagus nerve of one side only.

Two cases of bilateral palsy of the larynx with an aneurism pressing on one vagus and recurrent nerve have recently been recorded: one by Dr. Baümler, in the twenty-third volume of the 'Pathological Transactions;' the other by myself, in the twenty-fourth volume of the same Transactions. 
My own case ('Path. Trans.,' xxiv, p. 42) was that of a man forty-five years of age, who for several months had been suffering from stridulous and difficult breathing, the voice being feeble but tolerably clear. Looking into his larynx with the mirror, I saw the vocal cords of their natural colour, nearly touching each other in the middle line, and nearly motionless. There was a slight approximation of the cords during vocalisation. During inspiration the glottis did not expand as in the normal state, but, on the contrary, the cords appeared to be pressed nearer together by the inspiratory current of air, while in expiration again the cords were slightly pushed apart by the outgoing stream of air. No swelling or other structural change within the larynx was visible. There was dulness on percussion over the manubrium sterni-an impulse was heard there at each systole of the heart, and an impulse was felt by the ends of the fingers when firmly pressed against the bone. The patient stated that eighteen months before he had suddenly become hoarse while talking to a friend; the feebleness of voice had continued, and the breathing had gradually become difficult and attended with a noise in the throat. For some months past the breathing had become so difficult, especially when lying down at night, that he had been unable to sleep for more than a few minutes at a time. I came to the conclusion that the intrinsic muscles of the larynx on both sides were paralysed by the pressure of an aneurism of the aorta on one or both recurrent nerves.

During the first night after his admission into the hospital he got no sleep, and several times he appeared to be on the verge of suffocation. The following day Sir William Fergusson, at my request, performed tracheotomy. The operation afforded immediate and great relief, and the next night the patient slept for several hours. Two days afterwards symptoms of pleuro-pneumonia set in, and he died on the fourth day after the operation. An aneurism about the size of an orange projected backwards from the transverse aorta. The left vagus nerve passed in front of the aneurism, and was closely involved in its wall; the left recurrent passed 
round and behind the tumour, where it was compressed and atrophied, and nearly lost in the wall of the aneurism. There were some enlarged lymphatic glands near the right recurrent, but the nerve was not pressed upon or even touched by these glands, and both it and the right vagus appeared quite normal.

My friend and colleague Dr. Curnow did me the favour to dissect out the nerves and the muscles of the larynx, and he reported as follows:- "The laryngeal muscles on the left side are decidedly atrophied; those on the right side are somewhat larger, but I am inclined to think them atrophied also."

In Dr. Baümler's case ('Path. Trans.', xxiii, p. 66) with the physical signs of aneurism of the innominate artery there was dyspnœa with loud laryngeal stridor and aphonia. The laryngoscope showed complete immobility of the right vocal cord, with immobility almost as complete of the left cord. After death, which occurred from increasing dyspnœa, the right vagus was found flattened, and in one place almost lost in the wall of an aneurism of the innominata; the recurrent branch was also flattened and thinner than that on the left side. The left vagus and recurrent appeared perfectly normal everywhere. The intrinsic muscles of the larynx were very pale and flabby, and there was no appreciable difference in the bulk of the muscles on the two sides. The microscope showed granular degeneration of the muscles on both sides to an almost equal extent.

Dr. Baümler mentions first amongst the points of interest in this case "the absence of changes in the left recurrent, although during life the corresponding muscles showed, on laryngoscopic examination, very great impairment of their function."

In both these cases of aneurism the fact of bilateral palsy of the larynx was ascertained by a careful and thorough laryngoscopic examination during life; in both cases the laryngeal obstruction was greater than an affection of the

' The preparation is in the museum at King's College. 
muscles on one side only would account for. In both cases the post-mortem examination was made with the expectation of finding that the recurrent nerve on both sides had been injured, but in both instances it was found that the vagus and recurrent on one side only had been compressed; and, lastly, in both cases the intrinsic muscles of the larynx on both sides had undergone atrophic changes. In my own case the muscular atrophy was greater on the side of the compressed nerve, but in Dr. Baümler's case the wasting of the muscles appeared to be equal on the two sides.

For a long time the bilateral palsy of the larynx which unquestionably existed in these two cases appeared to me inexplicable, and I often discussed it with my physiological friends without obtaining a satisfactory explanation; but at length it occurred to me to suggest the following interpretation of the phenomena. ${ }^{1}$

The aneurism in each case compressed not only the recurrent branch, but also the trunk of the vagus; it seems, therefore, not improbable that while the muscles on one side of the larynx were paralysed by direct pressure on the recurrent branch on that side, the paralysis on the other side was the result of a centripetal irritation of the trunk of the vagus acting on the nervous centre, and through it upon the nerve-supply to the laryngeal muscles on the opposite side, so that the palsy on one side was direct, while that on the other side was the result of a reflex influence.

I now proceed to adduce in support of this theory such facts and arguments as $I$ have been able to gather from clinical observation, and from the results of experiments on living animals.

It is an unquestionable fact that the intrinsic muscles of the larynx are in a pre-eminent degree bilateral in their action. Every one who has inspected the living larynx knows how absolutely impossible it is in the normal condition to move one vocal cord without at the same time moving the other to an equal extent.

1 'British Medical Journal,' June 27th, 1874. 
According to Dr. Broadbent's ingenious and well-known hypothesis the muscles which thus act bilaterally must have their central nerve nuclei so closely connected by commissural fibres that the muscles of each side receive their nerve supply from both sides of the brain in proportion to the completeness of their bilateral action. The muscles of the larynx, which are in an especial degree bilateral in their action, in accordance with this hypothesis, must be on either side equally connected with both sides of the nervous centre. The central commissural connection between the nerves supplying the larynx is not a mere hypothesis or a physiological inference, but a demonstrated anatomical fact. Dr. Lockhart Clarke in his elaborate paper "On the Intimate Structure of the Brain"l has described and figured some of the fibres of origin of the spinal accessory nerve, decussating in three places across the median line of the medulla oblongata, and thus connecting the spinal accessory nuclei of the opposite sides. The spinal accessory nerve is known to be the source of the motor fibres in the laryngeal branches of the vagus, and in the structural arrangement thus demonstrated by Dr. Clarke, whereby the spinal accessory nuclei of the two sides are brought into close union with each other, we appear to have the explanation of the normal bilateral action of the laryngeal muscles. This commissural union of the nerve nuclei of bilaterally acting muscles explains certain wellknown pathological phenomena.

1st. As Dr. Broadbent has shown, it explains the fact, that in cases of hemiplegia resulting from a lesion of one hemisphere of the brain, the muscles which act bilaterally are not paralysed. The reason is, that although the motor influence from one side of the brain to these muscles is cut off or lessened, the motor influence from the other side reaches the commissural centre, and thence passes to the muscles on both sides. Dr. Broadbent explains it thus : "If the centre of volitional action on one side is destroyed, or one channel of motor power is cut across, the other will transmit 
an impulse to the motor centre, and this will be communicated to the nerves of the two sides; equally if the fusion of the two nuclei is complete, and there will be no paralysis; more or less imperfectly to the nerve of the affected side if the transverse connection between it and its fellow is not so perfect, in which case there will be a corresponding degree of paralysis."' The connection between the central origin of the nerves supplying the laryngeal muscles must be most intimate, for in cases of hemiplegia the result of a lesion of one cerebral hemisphere, however complete may be the palsy of the arm and leg, the muscles of the larynx appear to be quite unaffected by the paralysing influence. The voice, as a rule, is unchanged in these cases, and the laryngoscope shows an equally unimpeded movement of the cartilages and the vocal cords on both sides. In all cases of unilateral palsy of the laryngeal muscles the paralysing lesion must be below the junction of the nerve nuclei.

2nd. It is evident that the commissural union of the nerve nuclei, which explains the escape of bilaterally-acting muscles from palsy in cases of hemiplegia resulting from unilateral brain disease, renders these associated muscles liable to spasm when a morbid motor influence proceeds from either side of the nervous centre. Thus, in cases of hemispasmspasm, that is, of one arm and leg starting from a lesion of one cerebral hemisphere-the bilaterally-acting muscles of the chest and abdomen on both sides are liable to be, and are in fact, spasmodically affected; so the epileptic cry which is sometimes heard at the commencement of a convulsive attack is a result and a proof of bilateral spasm of the larynx. In these cases the morbid motor influence reaches the commissural fibres, and thence passes to the nerves and muscles on both sides. Thus the same commissural union which protects bilaterally-acting muscles from the paralysing influence of a one-sided brain-lesion, renders them liable to be implicated in convulsive attacks resulting from a disease on either side of the brain.

3rd. It is obvious that if the nerve nuclei and the com1 'British and Foreign Medico-Chirurgical Review,' April, 1866. 
missural fibres which connect them become diseased, bilateral palsy may result. And this is what probably happens as a result of a morbific influence conveyed to the nerve centres when the trunk of one vagus has been for a long time irritated by the pressure of an aneurism or other tumour.

Many cases are recorded, I have myself seen several, in which a tumour, aneurismal or cancerous, pressing on one recurrent nerve, has caused unilateral palsy of the intrinsic muscles of the larynx. The explanation of these cases is obvious. The conducting power of the efferent nerve is impaired or destroyed, and, as a necessary result, the muscles on that side are paralysed. It is equally obvious that pressure on one recurrent nerve alone, a nerve which is purely motor, and distributed only to the muscles on its own side, will not, either directly or indirectly, paralyse the muscles on the opposite side of the larynx. But it is, to say the least, extremely probable that pressure on the trunk of one vagus may, through an influence conveyed by its afferent fibres to the nerve centre produce bilateral palsy, as in the two cases whose histories I briefly gave at the commencement of this paper; while in other cases, to which I shall hereafter refer, bilateral spasm of the larynx may be excited through the same nervous channels.

It occurred to me, that some light might be thrown upon this subject by experiment; and my friend and former colleague, Professor Rutherford, did me the favour to perform some experiments on living rabbits, with results which I will now briefly describe. In all the experiments, the animal being fixed on its back, the larynx and trachea were exposed, and the anterior wall of the trachea just below the larynx was cut away, so that the glottis could readily be seen from below.

The superior laryngeal nerve was exposed and divided, and its central end was stimulated by a faradic current; the immediate result was a strong bilateral adduction of the vocal cords ; in other words, the glottis was spasmodically closed. This experiment was performed several times on four rabbits, and with uniform results. After we had performed this 
experiment, we found that the same results had before been obtained by other experimenters; in particular by Rosenthal, ${ }^{1}$ and by Waller and Prevost. ${ }^{2}$ The explanation is obviousa stimulus is sent through the afferent laryngeal nerve to the centre, and thence is reflected by the efferent fibres of the two vagi through the recurrent branches to the muscles of the larynx on both sides. This experiment then affords a good illustration of the physiological mechanism of bilateral spasm resulting from unilateral irritation.

The principle is probably the same, whether a morbid motor influence passes downwards from one side of the brain, or upwards through the afferent fibres of one vagus to the common centre of both vagi.

When the recurrent nerve on one side was stimulated, the vocal cord on the same side was abducted, while the opposite cord was unaffected.

Another experiment consisted in cutting across the trunk of the right vagus between the superior and the recurrent laryngeal nerves, and then applying the electrical stimulus to the lower end ; the result was abduction of the vocal cord on that side, while the cord on the opposite side remained motionless. Rosenthal found that this experiment performed on a cat caused unilateral adduction of the vocal cord. ${ }^{3}$ Whether abduction or adduction result from the stimulation of the recurrent or the distal end of the vagus depends probably upon the relative vigour of the antagonistic intrinsic muscles of the larynx.

When the central end of the right superior laryngeal was stimulated after the trunk of the vagus on the same side had been divided, the vocal cord on the opposite (left) side was adducted by the reflex nervous current, while the cord on the same side remained motionless in consequence of the efferent fibres in the trunk of the right vagus being cut across.

When the central end of the divided vagus is stimulated,

1 'Die Athembewegungen und ihre Beziehungen zum Nervus Vagus, Berlin, 1862, pp. 224, 225.

2 'Archives de Physiologie,' tome ii, 1870, p. 196.

${ }^{3}$ Loc. cit., pp. 211, 212. 
the result is not adduction of the opposite vocal cord, as when the superior laryngeal is irritated, but Rosenthal observed that the diaphragm and other inspiratory muscles are made to contract, and with this inspiratory action the vocal cord is abducted. ${ }^{1}$ Waller and Prevost also obtained the same results from the excitation of the central end of the vagus. ${ }^{2}$

These experiments serve to establish two general principles :

1st. Any movement of the larynx which results from stimulation of the afferent fibres or branches of one vagus is bilateral, except that, of course, division of the trunk of the vagus will prevent the occurrence of the reflex action on that side.

2nd. Stimulation of the efferent fibres of one vagus-the recurrent branch or the distal end of the divided trunkcauses movement on the one side only of the larynx.

In the course of our experiments we were enabled to verify an observation made long since by Longet, namely, that after the intrinsic muscles of the larynx have been paralysed by the division of all four laryngeal nerves, the glottis is closed by the compressing action of the inferior and middle constrictors of the pharynx during the act of deglutition. When after all the laryngeal nerves are divided the action of the constrictors of the pharynx has been suspended by the division of their fibres on one side, the glottis remains motionless during attempts at deglutition. Electrical stimulation of the central end of the superior laryngeal nerve always excites the act of deglutition, as Waller and Prevost have especially pointed out. We observed distinctly, as a result of such stimulation, that the vocal cords moved inwards and rapidly closed the glottis before the slower act of deglutition had commenced; but in two animals Dr. Rutherford divided the constrictors of the pharynx before the experiments on the superior laryngeal nerve were commenced, the object of this procedure being

1 Rosenthal, p. 214.

2 Loc. cit., p. 196.

3 'Archives Générales de Médecine,' tome xii, 1841, p. 423.

' 'Archives de Physiologie,' tome iii, 1870, p. 185. 
to enable us the better to distinguish the closure of the glottis by the constrictors of the pharynx during the act of deglutition from that which is caused by the intrinsic muscles of the larynx.

It is impossible by any experiment upon an animal to imitate all the influences which are in operation when an aneurism is gradually but constantly compressing the vagus and recurrent nerve in the human subject, but the results of the various experiments to which reference has been made give support and probability to the theory that a long-continued irritation of the trunk of one vagus may, through its afferent fibres, so disturb the common centre of the two vagi as to cause either bilateral spasm or bilateral palsy of the laryngeal muscles.

Reference may here be made to some well-known facts illustrating the influence of a peripheral irritation in exciting pathological changes in distant parts through a reflex nervous influence.

Cases of traumatic tetanus afford one good illustration of this. A foreign body in a wound may not only excite a general spasm of the muscles through an influence conveyed to the spinal cord and thence reflected through the efferent nerves to the muscles, but in fatal cases, as Dr. Lockhart Clarke has demonstrated, the spinal cord is found to have undergone extensive structural changes.

In the sixth volume of the "Transactions of the Clinical Society' I have published a case in which a piece of flint beneath the skin in a partially healed wound on the cheek excited facial neuralgia with facial palsy on the same side and trismus. These results of a reflex nervous influence all passed away after the removal of the foreign body which had unquestionably been their exciting cause.

During the passage of a renal calculus through the ureter there is often pain in the testicle on the same side and sometimes there is inflammatory swelling of the painful part. I extract the following from Sir James Paget's 'Surgical Pathology :'-“ Whoever has worked much with microscopes may have been conscious of some amount of inflammation of 
the conjunctiva in consequence of over-work. Now, the stimulus exciting this inflammation has been directly applied to the retina alone, and I have often had a slightly inflamed left conjunctiva after long working with the right eye while the left eye has been all the time closed. I know not how such an inflammation of the conjunctiva can be explained, except on the supposition that the excited state of the optic nerve is transferred or communicated to the filaments of the nerves of the conjunctiva, generating in them such a state as interferes with their nutrition."

It is a well-known fact that a foreign body lodged in one eyeball may, by a disturbing influence transmitted through the nerves and the nervous centre, excite destructive disease in the other eye; and it is a common and a successful practice to extirpate the primarily injured eyeball to prevent the induction of a secondary and sympathetic disease in its fellow.

The more intimate the nervous connection between two parts the greater is the probability that disease in one may excite sympathetic disorder in the other; the probability, then, is very great that irritation of the trunk of one vagus will gradually excite functional disorder, and even structural change, in the nervous centre and in the associated nerve on the opposite side.

Clinical observation has established the fact that laryngeal symptoms of some kind occur in a large proportion of cases of aneurism of the transverse aorta. Dr. Sibson has shown, in his elaborate analysis of the symptoms of aneurism of the aorta, that in cases of aneurism affecting the transverse portion of the arch the voice or cough was raucous or whispering, or inspiration was stridulous in $47 \cdot 5$ per cent. ${ }^{1}$

In the history of cases of aneurism symptoms are commonly attributed to pressure on the recurrent nerve, which could not possibly result from the implication of that nerve alone.

The recurrent, be it remembered, is an efferent motor nerve. When galvanized, as we saw, muscular contraction

$$
1 \text { 'Medical Anatomy,' Fasciculus } 5 .
$$


occurs only on the one side; no reflex bilateral contraction occurs, as when the afferent superior laryngeal nerve is stimulated. Pressure on one recurrent nerve may cause either unilateral spasm or unilateral palsy of the laryngeal muscles. Spasm from irritation of one recurrent would be indicated, as our experiments have shown, by abduction of the vocal cord from the median line, while a paralysed vocal cord remains motionless, or nearly so, in the median line during the act of inspiration. A unilateral palsy of the glottis may render the voice somewhat feeble, and it may cause some stridor on a deep inspiration, but it does not narrow the opening of the glottis sufficiently to seriously impede the breathing. I have seen a considerable number of cases in which the laryngoscope has shown unilateral palsy of the larynx, but in not one has distress of breathing been occasioned thereby. Dr. Bristow, in an interesting paper in the 'St. Thomas's Hospital Reports' (New Series, vol. iii, p. 205), refers to two cases in which unilateral palsy of the larynx was caused by the implication of one recurrent nerve in a cancerous tumour; but he states that in neither case "was there a trace of dyspnœa, either persistent or paroxysmal." The loud laryngeal stridor and the urgent dyspnœa which so frequently result from aneurisms and other tumours within the chest may be due to one of three conditions-lst, bilateral spasm of the laryngeal muscles, the result of irritation of one vagus; 2nd, bilateral palsy of the larynx, probably a later result of a tumour pressing on one vagus and recurrent; 3rd, palsy on one side, a direct result of pressure on the recurrent with spasm of the muscles on the opposite side, a reflex result of pressure on the trunk of the vagus.

When the dyspnœa and stridor are paroxysmal, coming on and going off with equal rapidity, the immediate cause is probably bilateral spasm of the larynx, and these attacks bear a striking resemblance to cases of laryngismus stridulus in children. On the other hand, long-continued laryngeal stridor and dyspnœa are commonly caused by paralysis of both vocal cords. The laryngoscope affords most valuable aid in the diagnosis of these interesting cases. 
It is probable that violent and prolonged spasm of the laryngeal muscles may sometimes be directly followed by a paralytic condition, during which the stridor and dyspnca continue, and death at length occurs from suffocation. This sequence of pathological events would seem to be analogous to the palsy which follows a fit of epilepsy, when the convulsed limbs are left paralysed. Dr. Hughlings Jackson ingeniously suggests that the violent physiological discharge of nerve-force through the motor fibres during the epileptic paroxysm may, for a time, suspend their functional activity, and thus cause the so-called " epileptic hemiplegia."

There is reason to believe that a bilateral affection of the larynx, either spasmodic or paralytic, is a very common result of pressure on the trunk of one vagus. The records of medicine abound with histories of cases in which death has resulted from laryngeal obstruction, and in which a postmortem examination has shown a tumour pressing on one vagus or on the vagus and its recurrent branch. I know of no case in which death has resulted from pressure on one recurrent nerve alone; but Dr. John Reid states that he has in his possession " a preparation procured from the body of a young man who died very suddenly, with all the symptoms of suffocation, when seated with some companions round a fire, who were chatting and laughing. Both recurrents are imbedded in a firm yellowish tumour, through which they cannot be traced."1

It is not to be supposed that a unilateral muscular affection of the larynx could cause suffocation. In all the fatal cases of this kind there must have been either a bilateral spasm or a bilateral palsy of the laryngeal muscles. The following cases may serve as illustrations of the principle for which I am here contending.

In the year 1847 I was asked by a medical friend to examine for him the body of a man, aged 29, who died after a six weeks' illness, from what was believed to have been laryngitis. The symptoms had been cough with scanty expectoration, hoarseness, noisy and difficult breathing, coming on

1 'Physiological, Anatomical, and Pathological Researches,' p. 275. 
in paroxysms, in one of which he died, notwithstanding an unsuccessful attempt to perform tracheotomy. This case occurred in the dark ages before the introduction of the laryngoscope, and the two experienced gentlemen who had attended the patient, one of them a general practitioner, the other a consulting surgeon, had no doubt that the case was one of laryngitis. We found after death a perfectly healthy larynx, but a cancerous tumour filled the concavity of the arch of the aorta and implicated the left vagus and recurrent. The right vagus and its branches were intact. In this pathological history we have the results of an experiment upon the vagus, and it can scarcely be doubted that the channel of nervous influence through which the cancerous tumour excited the fatal bilateral spasm of the larynx was the afferent fibres of the left vagus to the common centre, and thence through the efferent nerves to the muscles of the larynx on both sides.

In the 15th volume of the 'Pathological Transactions,' p. 72, I have published the case of a man, aged 31, who was admitted into the hospital with symptoms which were at first supposed to indicate laryngitis. There were hoarseness, cough, dyspnœa, and laryngeal stridor. When I first saw him, two days after his admission, the dyspnca and stridor had ceased, and I clearly saw, with the aid of the mirror, a healthy larynx with free movement of its cartilages and cords. The laryngeal symptoms which had been present, and a difficulty in swallowing solids, excited a suspicion of aneurism, although a careful examination detected no positive evidence of such disease. Two days later death occurred from hæmorrhage. An aneurism of the transverse aorta had compressed the left vagus and recurrent, and had opened into the cesophagus. In this case the pressure on the recurrent had not been sufficient to paralyse the muscles which it supplied, and it is believed that the laryngeal symptoms which had existed at the time of his admission were the result of bilateral spasm excited by the pressure of the aneurism on the trunk of the left vagus. ${ }^{1}$

1 The preparation is in the museum at King's College. 
On the 23rd of September in the present year $I$ saw, a few miles out of town, a gentleman, aged 40, who, for two months, had suffered from cough and dyspnœa, with stridulous inspiration. The superficial veins over both sides of the chest were enlarged, and the veins at the lower part of the neck were distended. There was dulness on percussion over the manubrium sterni, and a distinct systolic blowing was heard there. The stridor, on inspiration, was heard more distinctly on applying the stethoscope over the larynx than lower down over the trachea, and at the back over the spinous processes of the vertebræ it was more distinct in the middle cervical region, on a level with the larynx, than lower down over the upper dorsal spines opposite the arch of the aorta. The stridor, therefore, was the result of obstruction in the larynx, and not due to direct pressure on the trachea, such as occurred in a case of aortic aneurism which I once saw with my friend, Dr. Richards, of Winchester, and which I published in the 'British Medical Journal,' December 23rd, 1871, p. 720.

On proceeding to examine the larynx with the mirror, I found the fauces unusually sensitive, and not getting a complete view at once I discontinued the examination, expecting in a few days to have an opportunity to complete the investigation. Unfortunately, no such opportunity occurred. Two days after my visit the patient, who had been quietly talking to his wife, suddenly exclaimed, "I am being suffocated, help me." The breathing became more noisy and very difficult, the face was livid, the skin was soon bathed in a cold sweat, and in his agony he tossed about his arms and rolled on the floor. A neighbouring medical man, who was called in, gave him ether vapour, which appeared to relieve the extreme restlessness, but the breathing gradually became slower, consciousness was lost, and death occurred in about two hours after the sudden onset of the suffocating dyspncea. It is a remarkable fact that until he lost consciousness he was able to speak distinctly.

I regret extremely that I did not hear of the death until it was too late to make a post-mortem examination. In the 
absence of such an inspection some points of interest which might have been ascertained must remain doubtful.

I inferred from the symptoms and physical signs which I have described that an aneurism of the transverse aorta pressed on the superior cava, thus causing the venous fulness and on the left vagus causing the laryngeal symptoms. As the laryngeal stridor and dyspncea had continued with little change or variation for two months, it seemed probable that bilateral palsy of the muscles of the larynx was the cause of the obstructed breathing. Whether the final struggle, which began so suddenly, and ended so rapidly, was a result of a rapid increase of the laryngeal palsy or of a sudden spasm affecting the partially paralysed muscles, it is not possible now to decide.

Whatever may have been the immediate cause of the obstructed breathing, there can be no reasonable doubt that the seat of it was the larynx and not the trachea. It is probable, therefore, that the prompt performance ef tracheotomy might have prolonged life for a time.

This case, in some of its features, is very similar to the celebrated case of the Earl St. Maur, of which so interesting an account has been published by our President.

With reference to the question of tracheotomy in cases of aortic aneurism, obviously, the main point is to distinguish between laryngeal obstruction, the result of spasm or palsy caused by pressure on the vagus, and narrowing of the trachea by the direct pressure of an aneurism on the anterior wall of that tube. In the former class of cases tracheotomy may afford great temporary relief, as in the case recorded at the commencement of this paper, while in cases of obstruction of the trachea near its bifurcation, the operation would be worse than useless.

One fact which we observed in the course of our experiments is worthy of note on account of its bearing upon the therapeutics of laryngeal spasm. We found on two occasions that when a rabbit was under the influence of chloroform, the electric stimulus applied to the central end of the divided superior laryngeal nerve did not excite spasmodic 
closure of the glottis, although the slight respiratory movement of the cords continued. After the effect of the chloroform had passed away the same electrical stimulus caused the usual bilateral spasm of the glottis. Waller and Prevost also found that anæsthesia by ether or chloroform arrested the reflex movements which are ordinarily excited by the electrical stimulus applied to the central end of the superior laryngeal nerve in the cat. ${ }^{1}$ This antispasmodic effect of chloroform vapour is similar to the undoubted influence which the chloral hydrate exerts in preventing or lessening laryngeal spasm, whether in the purely spasmodic attacks of laryngismus stridulus or in cases of laryngitis with spasmodic complication, such as occurs very frequently in children and sometimes also in adults of both sexes.

The main points which $I$ have endeavoured to establish in this communication are the following :

1. When serious laryngeal dyspnœa results from the pressure of a tumour on the vagus and its branches, the immediate cause is a bilateral affection of the muscles of the larynx. There may be bilateral spasm or bilateral palsy or palsy on one side with temporary spasm of the muscles on the opposite side.

2. Pressure on one recurrent nerve can affect the laryngeal muscles on the one side only.

3. Pressure on the trunk of the vagus may through an influence upon the nervous centre cause either bilateral spasm or bilateral palsy of the larynx, and this is the true physiological interpretation of the urgent laryngeal symptoms which are often excited by the pressure of aneurismal and other tumours upon the vagus.

4. The bilateral spasm of the larynx which is excited by the pressure of a tumour on the trunk of one vagus is, probably, the result of a morbidly exalted excitability of the nervous centre induced by irritation of the afferent fibres of that nerve, and in consequence of this state of exalted sensibility ordinary stimuli, acting on the afferent nerves of the larynx, may cause bilateral spasm similar to that which is

1 'Archives de Physiologie,' tome iii, 1870, pp. 188, 189. 
excited by the extraordinary stimulus of electricity applied to the central end of the divided superior laryngeal nerve.

5. It is probable that the long-continued irritation of the trunk of the vagus may gradually, as in cases of traumatic tetanus, induce such demonstrable structural changes in the nerve-centre as will explain the bilateral palsy which appears to be one of the results of this chronic nerveirritation.

In any future cases of this kind that may occur and prove fatal after the laryngoscope has shown the existence of a bilateral palsy of the larynx, it will be desirable to subject the medulla oblongata and the nuclei of the spinal accessory and vagi nerves to a careful microscopic scrutiny. If the result of such an examination should be the discovery of structural changes in the nervous tissue the evidence in support of my theory will be complete.

Postscript.-Dr.Weir Mitchell, of Philadelphia, has recently done me the favour to send me a copy of a paper published by himself in conjunction with Dr. Morehouse. ${ }^{1}$ In this paper the authors describe a peculiar arrangement and distribution of the laryngeal nerves in the turtle, which appear to have an especial interest in relation to the subject of the preceding communication. In the turtle the movements of the glottis are effected by two pairs of muscles; by one pair of muscles the glottis is opened, while by another pair it is closed. The glottic muscles are supplied by two pairs of nerves, a superior laryngeal, and an inferior laryngeal or recurrent. The superior laryngeal nerves supply both the opening and the closing muscles of the glottis, while the inferior or recurrent laryngeal are distributed only to the opening muscles of the glottis. The superior laryngeal nerve, in addition to being the chief motor nerve of the larynx, in this respect being analogous to the recurrent in the higher animals, is proved by experiment to be the sensitive nerve of the larynx in the turtle. But the remarkable

1 "Researches upon the Anatomy and Physiology of Respiration in the Chelonia," 'Smithsonian Contributions to Knowledge,' Washington, 1863. 
arrangement which Drs. Mitchell and Morehouse have discovered consists in the existence of a true chiasm or decussation of fibres between the two superior laryngeal nerves, as a result of which each superior laryngeal nerve contains fibres from both sides of the nervous centre. This nervous chiasm resembles that of the optic nerves, and is, the authors believe, the only example of this arrangement yet observed in extracranial nerves. The authors were led to the discovery of this inter-communication between the superior laryngeal nerves by the results of experiment. They found that after both of the inferior laryngeal and one of the superior laryngeal nerves had been divided, the movements of the glottis continued unimpaired. They also found that Faradisation of the distal end of the divided superior nerve caused bilateral movement of the glottis. This result suggested the existence of some connection between the nerves of opposite sides. The inter-communicating fibres were then sought for and discovered in front of the larynx. It was found that after dividing the chiasm, division of one superior laryngeal nerve caused palsy of the glottic muscles on the same side. It thus appears that in the turtle the bilateral action of the laryngeal muscles is effected or assisted by such an inter-communication between the two principal motor nerves as gives to the muscles on either side an equal nerve-supply from both sides of the nervous centre. In the higher animals this union between the motor nerves of the larynx exists at their centre of origin.

Drs. Mitchell and Morehouse observed that after division of both inferior laryngeal nerves electric stimulation of the central end of one divided superior laryngeal nerve caused a reflex bilateral movement of the glottis. This shows that in the turtle, as in the higher animals, there is a central connection between the afferent fibres on one side and the efferent fibres on the other.

The correctness of the authors' conclusions as to the function of the laryngeal chiasm was confirmed by an experiment on one animal, which gave a result at first sight contradictory. 
In a large turtle, the two inferior laryngeal nerves having been divided, division of the right superior laryngeal caused palsy of the glottis on that side. This result was different from that previously obtained by the like experiment, and on a careful dissection it was found that the interlateral communicating fibres (the chiasm) were involved in a mass of diseased tissue, which had the same effect as division of the chiasm had been found to have in previous experiments. This observation, which at first appeared to throw doubt upon those which had preceded it, did, in fact, prove the correctness of the authors' previous conclusions. 